\title{
Compound micromachines powered by acoustic streaming
}

\author{
Murat Kaynak, Furkan Ayhan, and Mahmut Selman Sakar
}

\begin{abstract}
This paper presents the design, fabrication, and operation of compound micromachines powered by acoustic streaming. The machine components were directly incorporated around pillars serving as shafts without further assembly steps using a single-step in situ polymerization process controlled by a programmable projector. Two strategies were presented for harvesting acoustic energy using sharp-edged structures. The first method is based on on-board pumping of fluids and the second method involves engineering of rotors. The implementation of these strategies resulted in the construction of microscale turbines and engines that can be coupled to gear trains for adaptable transmission of mechanical power. We provide a number of further improvements that may together lead to development of compact yet powerful robotic manipulation systems inside microfluidic devices.
\end{abstract}

\section{INTRODUCTION}

Microfluidic laboratory-on-a-chip technology promises to enable automated execution of macroscale, bench-top experimental protocols in low-cost, miniaturized devices [1], [2]. Precise manipulation of small volumes of fluids and biological samples along with the ability to perform quantitative analysis has already started to revolutionize clinical diagnostics and pharmaceutical research [3]. While sensing and visualization techniques have been rapidly advancing [4], actuated structures are largely limited to the use of conventional pneumatic valves [5]. Previous works demonstrated the feasibility of operating remotely controlled robotic microtools inside microfluidic chips for micromanipulation [6]-[15]. These untethered simple machines provide spatial control over mass transport, yet the design and fabrication methodology is not appropriate for the construction of modular and highly customizable, complex integrated microsystems.

A simple and cost-effective method to overcome viscous forces and drive microscopic machinery inside microfluidic channels is the use of sound waves generated by piezoelectric transducers [16]-[19]. Microbubbles whose surfaces oscillate when forced with acoustic waves generate powerful flows that can be harnessed for microscale propulsion [20], [21]. While bubble cavitation is the oldest and most extensively studied method for utilizing acoustic power in biomanipulation [22], there are a number of limitations for precision engineering applications. Encapsulation of bubbles exclusively inside engraved cavities on driver components is a challenging procedure. Furthermore, there is no reliable method for retaining the size and shape of the bubbles during long-term continuous excitation inside biological fluids at

The authors are with the Institute of Mechanical Engineering, Ecole Polytechnique Federale de Lausanne, Lausanne, 1015, Switzerland. (email: murat.kaynak@epfl.ch, furkan.ayhan@epfl.ch,selman.sakar@epfl.ch)
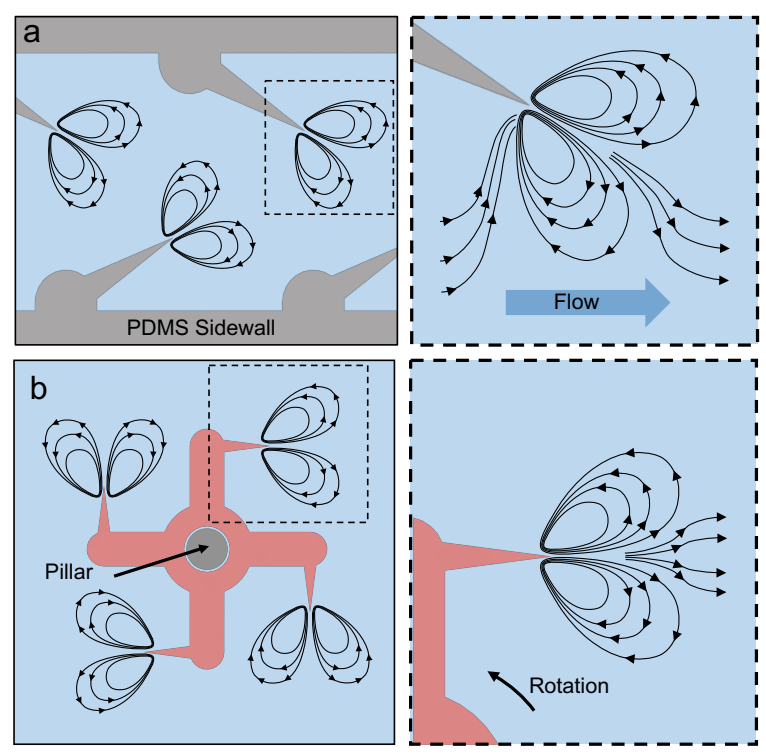

Fig. 1. Schematic depiction of working principles for machines powered by acoustic streaming. Upon application of acoustic waves, sharp-edged structures oscillate and generate counter rotating vortices. These vortices can be utilized to drive machinery by generating (a) fluid flow inside a watermill system and (b) rotation of engines around a shaft (not to the scale).

physiological temperatures. Solid bodies vibrating in fluids also generate steady streaming if they have sharp features [23]-[25], providing a more robust and scalable solution for acoustofluidic actuation. Building devices decorated with sharp-edged structures to drive multi-body robotic microsystems is the focus of this work.

We present two distinct strategies for powering compound micromachines (Figure 1). In the first mechanism, the the walls of the microchannel are decorated with actuated structures to generate pressure difference and drive a turbine located downstream of the pumping area (Figure 1a). The second mechanism is driven by a rotor generating acoustic streaming with sharp features attached to its body (Figure 1b). Microscale posts fabricated inside the channels serve as shafts for rotating active and passive elements. Maskless optofluidic photopolymerization process enables the manufacturing of custom shaped functional microparts, which we use to create compound micromachines without requiring further assembly. The blueprint for the chassis and machine components are highly re-configurable, which allows rapid design and prototyping of a variety of different systems. We provide guidelines for constructing more sophisticated systems with additional mechanisms such as a clutch. 

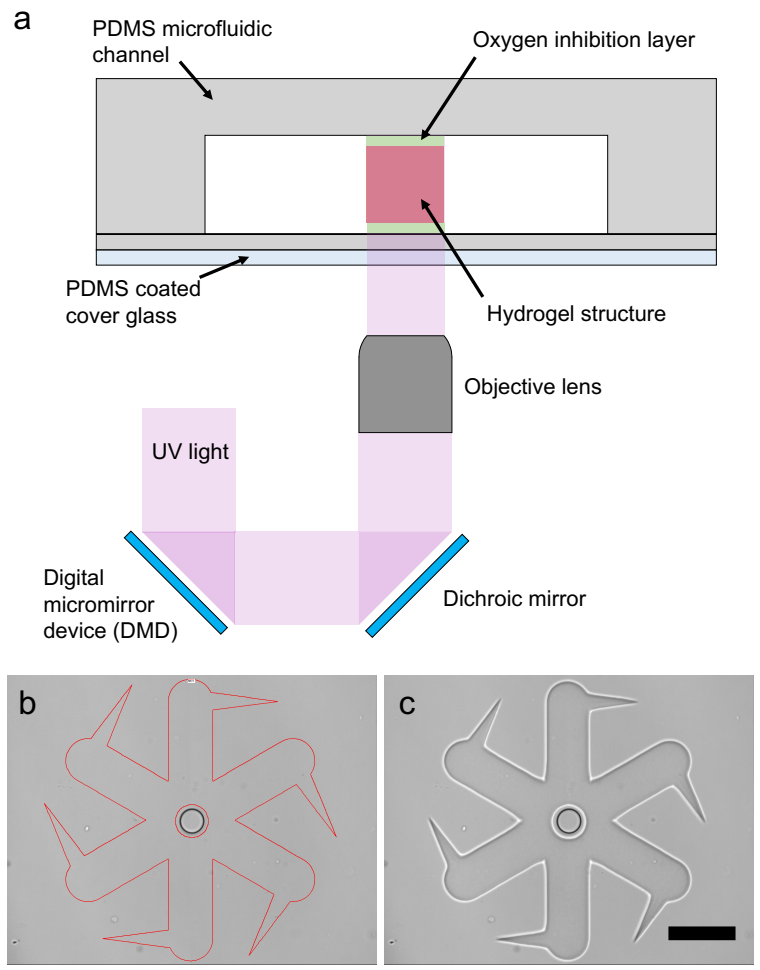

Fig. 2. Fabrication of hydrogel micromachines using digital maskless photolithography inside microchannels. (a) Schematic illustration of the fabrication process. UV light is patterned and focused by a programmable microscope system according to the CAD drawings. Oxygen inhibition layer permits mobilization of structures. (b) The light pattern projected on the screen and (c) the microstructure polymerized at the end of the exposure process. Scale bar, $250 \mu \mathrm{m}$.

\section{EXPERIMENTAL METHODS}

\section{A. Development of the Micromachinery}

The systematic assembly of microfabricated parts and integration of assembled mechanisms with microfluidic devices are extremely complex tasks. In situ polymerization of photocurable polymers directly inside closed chambers is a promising alternative for rapid prototyping [26]. Hydrogels are particularly interesting materials for building machinery due to their tunable mechanical properties and stimuli-responsive behavior [27]. Figure 2a illustrates the fabrication of hydrogel components around existing polydimethylsiloxane (PDMS) pillars inside microfluidic channels. Microfluidic devices were fabricated using replica molding. In this work, channel height was set to be $100 \mu \mathrm{m}$. Sharp features required for the development of the pumping regions demand for high-resolution photolithography. We wrote the molds on chrome masks using a laser writer (VPG 200, Heidelberg) with $600 \mathrm{~nm}$ resolution to address this requirement. After development, the molds were coated with Trichloro $(1 \mathrm{H}, 1 \mathrm{H}, 2 \mathrm{H}, 2 \mathrm{H}$-perfluorooctyl) to facilitate the removal of the PDMS devices.

PDMS is a gas permeable material and an oxygen layer (on the order of $1 \mu \mathrm{m}$ ) forms on the surface of the devices [28] during plasma treatment. This layer inhibits polymer-

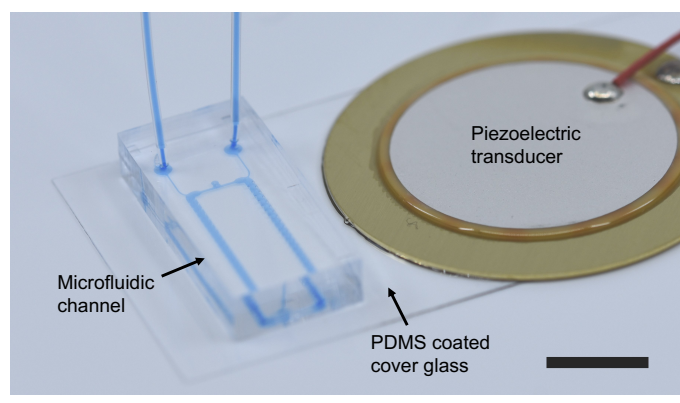

Fig. 3. Image of the integrated device consisting of the microfluidic channels and the piezoelectric transducer, all bonded to a glass slide. The acoustic waves are transmitted to the microfabricated machinery through the glass substrate. Scale bar, $10 \mathrm{~mm}$.

ization close to walls, which is instrumental for constructing free-floating machinery that are not attached or physically interacting with the boundaries. The glass slide, on which the microfluidic device and the piezoelectric transducer were attached, was coated with a thin layer of PDMS to utilize this lubrication strategy. As a further precaution, components were fabricated with bores slightly larger than the pillars. This approach ensures the free rotation of microcomponents around the shaft at all times. We used a low pressure syringe pump (neMESYS 290N, CETONI) to introduce the photocurable prepolymer solution $(50 \%(\mathrm{v} / \mathrm{v})$ polyethylene glycol diacrylate (PEGDA) 700, 30\% (v/v) PEGDA 250, $20 \%$ (v/v) photoinitiator 2-Hydroxy-2-methylpropiophenone (Darocur 1173). All reagents were bought from Sigma Aldrich and used according to the manufacturer's instructions. Polymerization pattern during flow lithography was controlled by a programmable digital micromirror device (MM-8201-801, Andor). The projected UV light was focused onto the bottom layer of microchannels through a $10 \mathrm{x}$ objective lens (Plan Flour 10X/0.3 DIC L/N1, infinity/0.17 WD 16.0, Nikon). The intensity of UV light on the sample $\left(80 \mathrm{~mW} / \mathrm{cm}^{2}\right)$ was measured using an optical power meter (PM400, Thorlabs).

\section{B. Acoustic Control System}

The sinusoidal control signals were generated by a programmable function generator (AF-2225, GW Instek), which were amplified by a power amplifier (TOE 7610, TEOLLNER) before reaching the piezoelectric transducer (81-7BB27-4LO, Murata Electronics). The transducer was bonded onto the glass slide using 5-minute epoxy (G14250, Devcon). The transducer was located in the center of the glass slide to enhance vibrations during operations on the microscope stage. The frequency and amplitude of input voltage were monitored using an oscilloscope (TBS 1032B, Textronix) during experiments. Experiments were performed with an inverted fluorescence microscope (Ti2, Nikon) and videos were recorded using a high-speed camera (VEO640L, Phantom).

\section{RESULTS}

Sharp edges generate oscillations at their tips, resulting in a pair of counter rotating vortices. There are pushing 

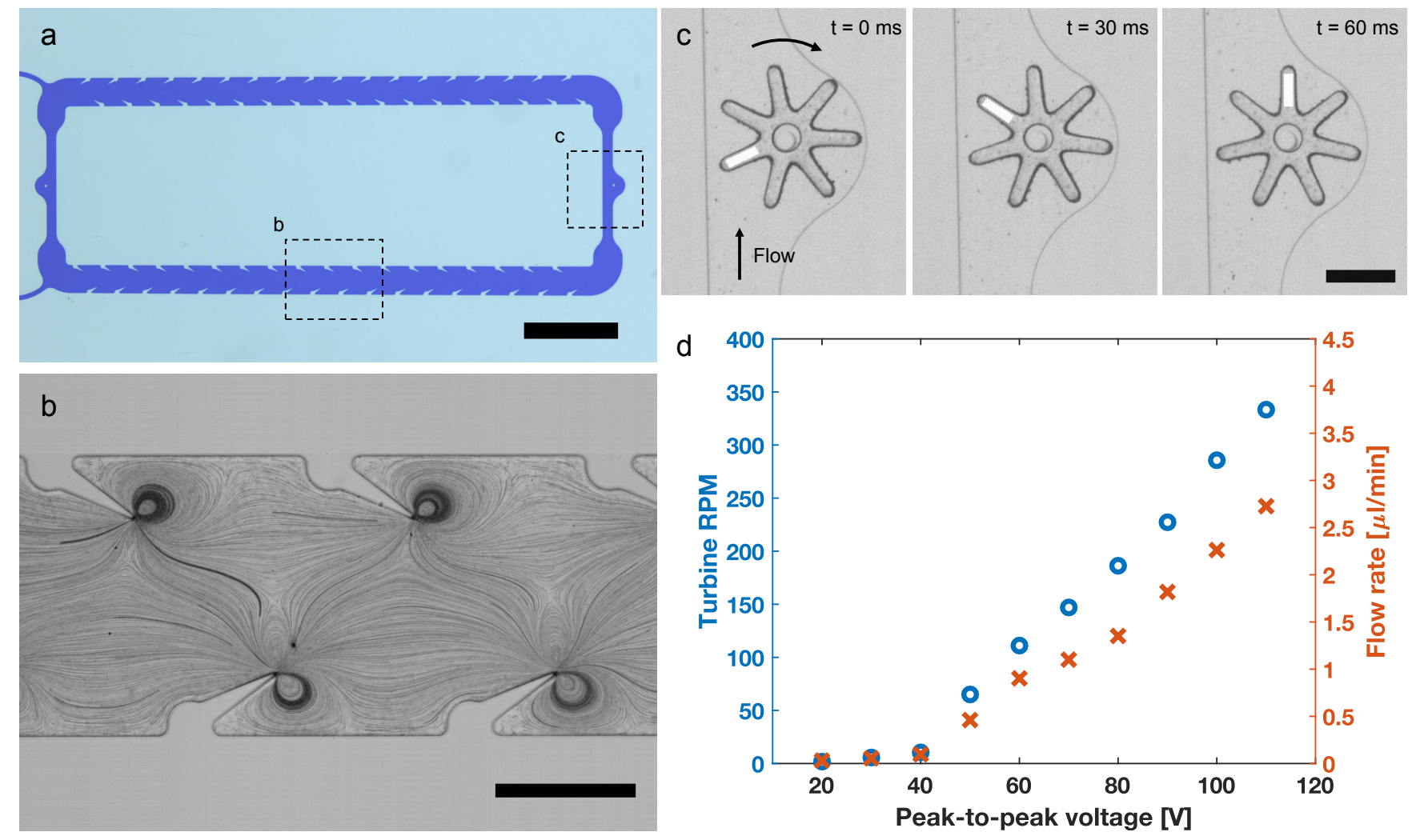

Fig. 4. The design and operation of acoustically powered microturbines. (a) A representative image of the system designed to pump fluids along the closed hydraulic network and rotate the turbine. Scale bar, $2 \mathrm{~mm}$. (b) The fluid flow generated in the pumping area, visualized by particle tracking. Scale bar, $300 \mu \mathrm{m}$. (c) The sequence of images from a rotating turbine. The white bars indicate specific teeth locations. Arrows show direction of rotation and flow. Scale bar, $200 \mu \mathrm{m}$. (d) A representative plot of flow rate and turbine revolution rate as a function of the input voltage.

and pulling forces generated at each oscillation cycle. The displacement of fluid during pushing is higher than the pulling phase due to viscous attenuation, resulting in pressure fluctuations and formation of a localized jet. We utilized this phenomena in two different ways to power microscale autonomous devices.

\section{A. Microturbines driven by acoustic pumping}

Figure 4a shows an image of the device, revealing the overall architecture of the waterwheel mechanism. The fluid flow generated by the sharp-edged structures on the walls of the microchannel is channeled through a closed hydraulics network. The structures were placed bilaterally throughout the pumping area (Figure 4b) with a spacing and angle optimized for pumping efficiency [25]. The structures were expected to provide maximum acoustic streaming at the resonant frequency of the transducer $(4.6 \pm 0.6 \mathrm{kHz})$. However, at this frequency, geometrical deviations from a straight profile such as curved channels or structural impurities generate non-linear effects that interfere with the flow. We performed a frequency sweep test between 1 and $100 \mathrm{kHz}$ and measured the corresponding flow rate. The best performance was recorded at $30.9 \pm 1 \mathrm{kHz}$ and, thus, this frequency was chosen as the driving frequency. We injected polystyrene microparticles with a diameter of 1 micron into the microchannel to visualize the fluid flow. Vortices are expected to form symmetrically when the tip of the sharp edge is normal to the side wall. By tilting the structures by $30^{\circ}$, we modulated the distribution of vortices and, as a result, created net flow that was observable through the streamlines (Figure 4b).

The turbine was formed around the pillar that was engineered inside a circular confinement downstream the pumping area (Figure 4c). The primary consideration here is that at low Reynolds number viscous fluids obey the noslip condition. The thickness of the blades of the turbine was optimized for effective transmission of hydrodynamic forces. Low thickness led to bending of the blades while high thickness generated large viscous drag. The angular speed of the turbine was controlled by tuning the peak-to-peak voltage $\left(\mathrm{V}_{\mathrm{PP}}\right)$ that was applied to the piezoelectric transducer. As a rule of thumb, the higher the input voltage the larger the flow rate due to increase in vibration amplitude. The hydrodynamic forces started to exceed the drag forces and reached enough power to rotate the turbine at $20 \mathrm{~V}_{\mathrm{PP}}$ (Figure $4 d)$. The angular speed increased with applied voltage for all tested values. The rotation completely halted within $\approx 2 \mathrm{~ms}$ after turning off the input source due to the characteristics of low Reynolds number flow.

\section{B. Computational analysis of acoustic streaming}

We performed numerical simulations of the pump mechanism using COMSOL Multiphysics software. We first identi- 


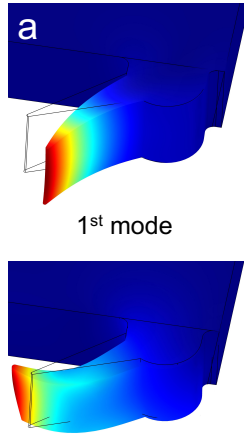

$3^{\text {rd }}$ mode

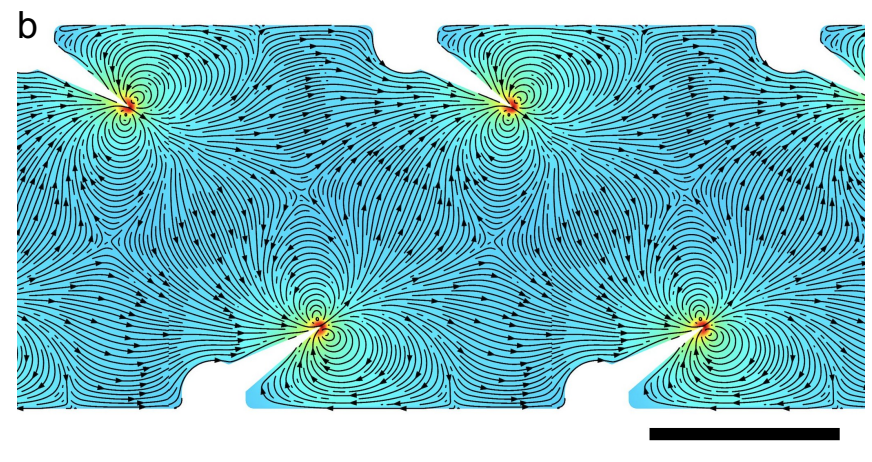

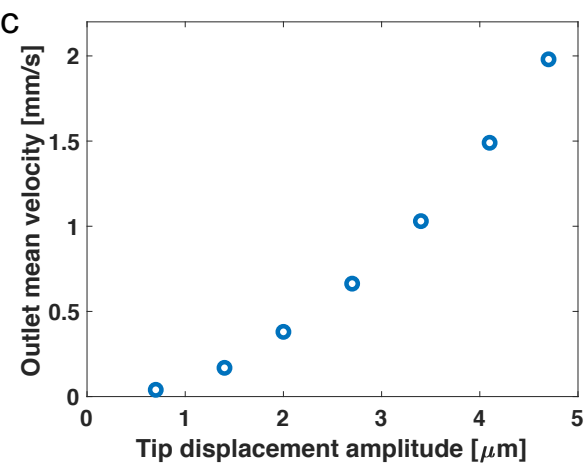

Fig. 5. Simulation results. (a) The dominant vibration modes of the structures at $27 \mathrm{kHz}$. Color represents relative amplitude. (b) The streamlines generated inside the pumping area during excitation at $27 \mathrm{kHz}$. The mean velocity is computed on the fluid domain over 10 oscillation cycles. Scale bar, $300 \mu \mathrm{m}$. (c) The mean velocity along the long axis of the channel at the outlet that is calculated at different displacement amplitudes.

fied the eigenfrequencies of the structures by running coupled acoustic-structure finite element simulations. The oscillating structures are defined as linear elastic materials due to the mechanical properties of the elastomer. After investigating the vibration modes corresponding to different resonant frequencies, we concluded that the best pumping performance is expected at $6.5 \mathrm{kHz}$ and $27 \mathrm{kHz}$. The eigenmodes that are driving the vibrations at $27 \mathrm{kHz}$ are shown in Figure 5a. The slight increase in the resonance frequency compared to empirical results can be due to the damping in the system and the losses at the interfaces, which were neglected in the computational model. We also assumed that the propagation of the acoustic wave in the fluid does not influence the excitation of the structures because the wavelength of sound in fluid is significantly larger than the characteristic length of the oscillating structures.

In the second part, we developed a computational fluid dynamics model to study the flow generated inside the channel using a one-way fluid-structure coupling scheme. That is to say, the actuated structures influence the surrounding fluid but the fluid does not effect the motion of the structure. We applied periodic displacements to the sharp-edged structures according to the excited eigenmodes. Figure $5 \mathrm{~b}$ shows the streamlines generated by the oscillating structures at $27 \mathrm{kHz}$ where we computed the mean velocity over 10 oscillation cycles. We assumed that all structures were actuated with the same acoustic field, which led to a symmetrical flow field inside the channel. We observed asymmetry in the experimentally recorded flow field (Figure $4 b$ ) due to the damping of pressure waves during power transmission. We modified the amplitude of the oscillations to derive a relationship between the flow rate in the channel and the input power. The net flow rate increases with the increasing amplitude after exceeding a certain threshold and the trend seems to be linear (Figure 5c). The mean velocity was measured as 0.8 $\mathrm{mm} / \mathrm{s}$ which corresponds to a numerical oscillation amplitude of $3 \mu \mathrm{m}$ and we verified that the tip movement during experiments corroborates with this prediction. Interestingly, simulations also showed that the streamlines do not depend on the amplitude of the oscillations for a given frequency.
The computational framework can be used as a design tool to investigate the influence of device parameters such as the distribution and geometry of structures, channel dimensions, and material properties.

\section{Microengines powered by acoustic rotors}

An alternative paradigm for driving microsystems is having an on-board mechanism to directly harvest acoustofluidic power (Figure 6). The pointy extensions fabricated on the rotor arms generated coordinated acoustic streaming, which induced torque on the rotor. Rotors with multiple arms, all equipped with sharp edges, were expected to generate higher angular speed. On one side, we would like to move the sharp edges as far from the tip of the arm as possible to protect the rest of the machinery from the generated fluid flow. On the other side, confinement of sharp edges between the arms significantly impedes the steady flow. Therefore, we engineered long and short arms, to address both limitations. Together with the gear, the rotor formed an acoustically controlled engine. While long arms were interacting with the gear to transmit power, there was ample space left around the sharp edges for maximizing torque. The width of the arms also played an important role in the performance. We designed $500 \mu \mathrm{m} \times 100 \mu \mathrm{m}$ arms because these dimensions resulted in maximum speed.

The speed of rotors increased quadratically with input voltage. This relation could be explained by the power relation of the transducer. Two different regimes were observed during amplitude modulation. At low voltage, the power transmitted by the rotor was not sufficient to rotate the gear. The gear started to rotate at $60 \mathrm{~V}_{\mathrm{PP}}$ and its speed monotonically increased with input voltage. The gears were designed for optimal coupling with the rotor. The ratio between the angular speed of the rotor and the gear was given by $1: 2$, which is close to the ratio of the number of arms (3:7). The small discrepancy can be explained by imperfect coupling between the arms during rotation and structural defects on the parts. The existence of a chamber around the gear serves the same purpose as the chamber around the turbine, minimization of secondary flows. Although these structures did not have 

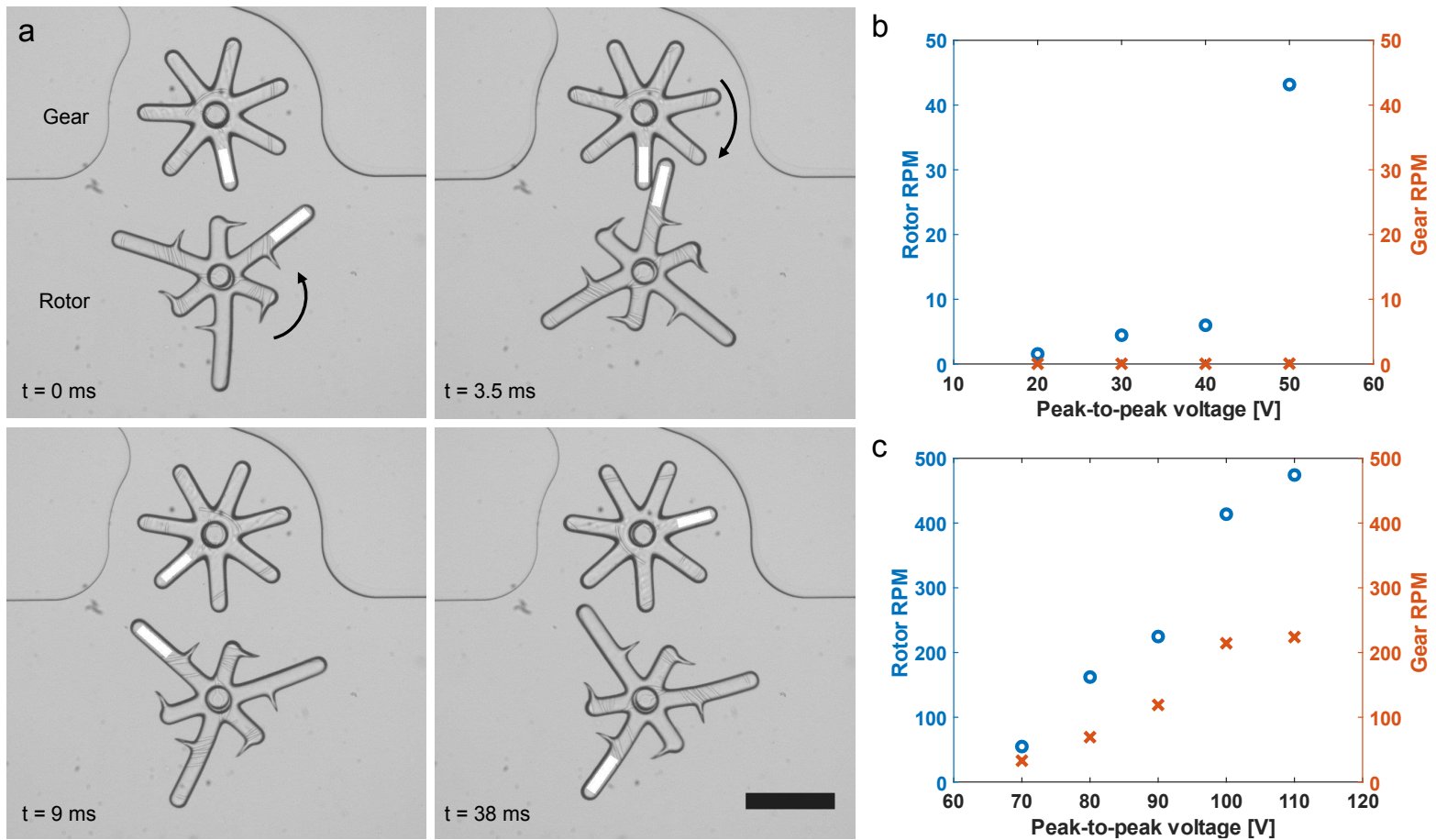

Fig. 6. The design and operation of acoustically powered microengines. (a) An image series of a rotating rotor and gear pair. While the rotor continuously moves, the gear display intermittent rotation due to the mismatch between teeth number. The white bars indicate specific teeth locations and the arrows show the direction of motion. Scale bar, $400 \mu \mathrm{m}$. Representative plot of the revolution rate for the rotor and the gear (b) at low and (c) high input voltages.

sharp features, they could still generate vortices around the tip of the arms. The walls surrounding the structures prevented completion of the formation of streamlines.

\section{Construction of transmission}

The speed ratio and the mechanical advantage of the systems can be systematically controlled by the incorporation of multiple gears (Figure 7). We tested whether we could add another gear without significantly reducing the power output of the watermill system (Figure 7a). The engaged turbinegear pair had consistently lower angular speed than the single turbine as expected. Likewise, incorporating another gear to form a gear train into the microengine resulted in reduction in output torque (Figure $7 \mathrm{~b}$ ). The gears were rotating synchronously and leak flows did not have a significant effect on angular speed. We also engineered gears with different number of teeth. The measured value of the speed ratio for the pair of meshing gears were the same as the ratio of the number of teeth on each gear for all input voltage values, as expected.

\section{DISCUSSION}

The parts for building a transmission is not limited to the use of gears. The power output of the systems can be modulated by disengaging the turbine or the rotor from the transmission using a clutch. The presented fabrication methodology is compatible with stimuli responsive materials such as poly(N-isopropylacrylamide) pNIPAM. The swelling degree of pNIPAM depends on the temperature, which can be remotely modulated by light with the incorporation of

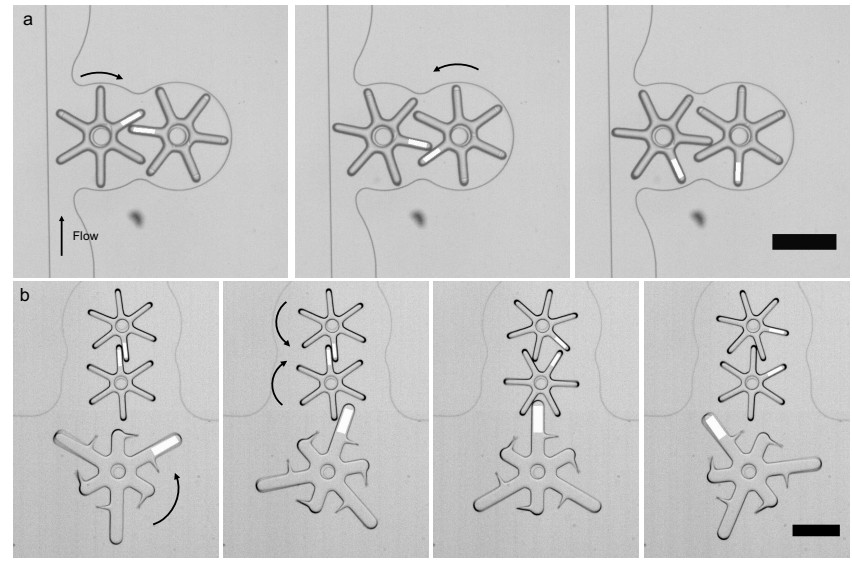

Fig. 7. The design and operation of gear trains. An image series demonstrating torque transmission in the (a) watermill system and (b) microengine. The white bars indicate specific teeth locations and arrows denote the direction of rotation. Scale bars, $400 \mu \mathrm{m}$ and $300 \mu \mathrm{m}$.

magnetic or gold nanoparticles [29], [30]. The reduction in size can be utilized to reversibly disconnect the driving part from the rest of the mechanism. Another upgrade would be to engineer a rack and pinion type mechanism to convert rotational motion into linear motion. The rack can be photopolymerized directly inside a microchannel next to the rotary engine in order to constrict the translational motion to a line.

We do not currently have control over the direction of rotation of neither the turbines nor the engines because 
sharp-edged structures generate unidirectional flow. Optical modulation of the shape of the structures may address this issue through the modulation of the alignment of sharp edges. By re-aligning the direction the tips, the streamlines may be dynamically moved. Alternatively, multiple sharp features with varying shapes or mechanical properties can be engineered on the same body to selectively excite acoustic streaming at different locations with the aid of frequency modulation. A similar approach has been recently employed to selectively actuate microbubbles that have unique dimensions [20]. This strategy may also lead to coordinated operation of multiple rotors on the same device.

In addition to providing control over power transmission, gear trains can also serve for another important purpose. The sharp edges generate secondary flows that are strong enough to dominate the motion of the machinery. However, there are primary flows generated by the propagating acoustic waves in the medium and, especially at high voltages, these flows may interfere with the intended operation inside the microfluidic device. The transmission may allow complete decoupling of the end-effector from the driving mechanism and provide a flow free arena for the manipulation tasks. Furthermore, with the incorporation of clutch mechanisms, multiple different tasks may be completed by the same driving mechanism.

\section{ACKNOWLEDGMENT}

We gratefully acknowledge financial support from Ecole Polytechnique Federale de Lausanne (EPFL) and Turkey's Ministry of Education. We would like to thank Pietro Dirix for his assistance with the computational analysis of the system. We would also like to thank the personnel working at the Center of MicroNanotechnology and the members of MICROBS laboratory for their technical support.

\section{REFERENCES}

[1] G. M. Whitesides, "The origins and the future of microfluidics," Nature, vol. 442, no. 7101, p. 368, 2006.

[2] P. Yager, T. Edwards, E. Fu, K. Helton, K. Nelson, M. R. Tam, and B. H. Weigl, "Microfluidic diagnostic technologies for global public health," Nature, vol. 442, no. 7101, p. 412, 2006.

[3] E. K. Sackmann, A. L. Fulton, and D. J. Beebe, "The present and future role of microfluidics in biomedical research," Nature, vol. 507, no. 7491, p. 181, 2014.

[4] S. K. Vashist, P. B. Luppa, L. Y. Yeo, A. Ozcan, and J. H. Luong, "Emerging technologies for next-generation point-of-care testing," Trends in biotechnology, vol. 33, no. 11, pp. 692-705, 2015.

[5] M. A. Unger, H.-P. Chou, T. Thorsen, A. Scherer, and S. R. Quake, "Monolithic microfabricated valves and pumps by multilayer soft lithography," Science, vol. 288, no. 5463, pp. 113-116, 2000.

[6] M. Boukallel, M. Gauthier, M. Dauge, E. Piat, and J. Abadie, "Smart microrobots for mechanical cell characterization and cell convoying," IEEE transactions on biomedical engineering, vol. 54, no. 8, pp. 15361540, 2007.

[7] L. Zhang, K. E. Peyer, and B. J. Nelson, "Artificial bacterial flagella for micromanipulation," Lab on a Chip, vol. 10, no. 17, pp. 2203$2215,2010$.

[8] W. Hu, K. S. Ishii, and A. T. Ohta, "Micro-assembly using optically controlled bubble microrobots," Applied Physics Letters, vol. 99, no. 9, p. 094103, 2011.

[9] T. Kawahara, M. Sugita, M. Hagiwara, F. Arai, H. Kawano, I. ShihiraIshikawa, and A. Miyawaki, "On-chip microrobot for investigating the response of aquatic microorganisms to mechanical stimulation," $L a b$ on a Chip, vol. 13, no. 6, pp. 1070-1078, 2013.
[10] E. B. Steager, M. Selman Sakar, C. Magee, M. Kennedy, A. Cowley, and V. Kumar, "Automated biomanipulation of single cells using magnetic microrobots," The International Journal of Robotics Research, vol. 32, no. 3, pp. 346-359, 2013.

[11] H.-W. Tung, D. F. Sargent, and B. J. Nelson, "Protein crystal harvesting using the rodbot: a wireless mobile microrobot," Journal of Applied Crystallography, vol. 47, no. 2, pp. 692-700, 2014.

[12] Z. Ye and M. Sitti, "Dynamic trapping and two-dimensional transport of swimming microorganisms using a rotating magnetic microrobot," Lab on a Chip, vol. 14, no. 13, pp. 2177-2182, 2014.

[13] T.-Y. Huang, M. S. Sakar, A. Mao, A. J. Petruska, F. Qiu, X.-B. Chen, S. Kennedy, D. Mooney, and B. J. Nelson, "3d printed microtransporters: Compound micromachines for spatiotemporally controlled delivery of therapeutic agents," Advanced Materials, vol. 27, no. 42, pp. 6644-6650, 2015.

[14] W. Jing, S. Chowdhury, M. Guix, J. Wang, Z. An, B. V. Johnson, and D. J. Cappelleri, "A microforce-sensing mobile microrobot for automated micromanipulation tasks," IEEE Transactions on Automation Science and Engineering, pp. 1-13, 2018.

[15] J. Yu, B. Wang, X. Du, Q. Wang, and L. Zhang, "Ultra-extensible ribbon-like magnetic microswarm," Nature communications, vol. 9, no. 1, p. 3260, 2018.

[16] M. Kaynak, A. Ozcelik, N. Nama, A. Nourhani, P. E. Lammert, V. H. Crespi, and T. J. Huang, "Acoustofluidic actuation of in situ fabricated microrotors," Lab on a Chip, vol. 16, no. 18, pp. 3532-3537, 2016.

[17] N. Bertin, T. A. Spelman, T. Combriat, H. Hue, O. Stéphan, E. Lauga, and P. Marmottant, "Bubble-based acoustic micropropulsors: active surfaces and mixers," Lab on a Chip, vol. 17, no. 8, pp. 1515-1528, 2017.

[18] D. Ahmed, A. Ozcelik, N. Bojanala, N. Nama, A. Upadhyay, Y. Chen, W. Hanna-Rose, and T. J. Huang, "Rotational manipulation of single cells and organisms using acoustic waves," Nature communications, vol. 7, p. $11085,2016$.

[19] A. Ozcelik, N. Nama, P.-H. Huang, M. Kaynak, M. R. McReynolds, W. Hanna-Rose, and T. J. Huang, "Acoustofluidic rotational manipulation of cells and organisms using oscillating solid structures," Small, vol. 12, no. 37, pp. 5120-5125, 2016.

[20] D. Ahmed, M. Lu, A. Nourhani, P. E. Lammert, Z. Stratton, H. S. Muddana, V. H. Crespi, and T. J. Huang, "Selectively manipulable acoustic-powered microswimmers," Scientific reports, vol. 5, p. 9744, 2015.

[21] N. Bertin, T. A. Spelman, O. Stephan, L. Gredy, M. Bouriau, E. Lauga, and P. Marmottant, "Propulsion of bubble-based acoustic microswimmers," Physical Review Applied, vol. 4, no. 6, p. 064012, 2015.

[22] P. Marmottant and S. Hilgenfeldt, "Controlled vesicle deformation and lysis by single oscillating bubbles," Nature, vol. 423, no. 6936, p. 153, 2003.

[23] M. Ovchinnikov, J. Zhou, and S. Yalamanchili, "Acoustic streaming of a sharp edge," The Journal of the Acoustical Society of America, vol. 136, no. 1, pp. 22-29, 2014.

[24] M. Kaynak, A. Ozcelik, A. Nourhani, P. E. Lammert, V. H. Crespi, and T. J. Huang, "Acoustic actuation of bioinspired microswimmers," Lab on a Chip, vol. 17, no. 3, pp. 395-400, 2017.

[25] P.-H. Huang, N. Nama, Z. Mao, P. Li, J. Rufo, Y. Chen, Y. Xie, C.H. Wei, L. Wang, and T. J. Huang, "A reliable and programmable acoustofluidic pump powered by oscillating sharp-edge structures," Lab on a Chip, vol. 14, no. 22, pp. 4319-4323, 2014.

[26] S. E. Chung, W. Park, H. Park, K. Yu, N. Park, and S. Kwon, "Optofluidic maskless lithography system for real-time synthesis of photopolymerized microstructures in microfluidic channels," Applied Physics Letters, vol. 91, no. 4, p. 041106, 2007.

[27] H. Lee, J. Kim, J. Kim, S. E. Chung, S.-E. Choi, and S. Kwon, "Programming magnetic anisotropy in polymeric microactuators," Nature materials, vol. 10, no. 10, p. 747, 2011.

[28] A. C. Urness, E. D. Moore, K. K. Kamysiak, M. C. Cole, and R. R. McLeod, "Liquid deposition photolithography for submicrometer resolution three-dimensional index structuring with large throughput," Light: Science \& Applications, vol. 2, no. 3, p. e56, 2013.

[29] H.-W. Huang, M. S. Sakar, A. J. Petruska, S. Pané, and B. J. Nelson, "Soft micromachines with programmable motility and morphology," Nature communications, vol. 7, p. 12263, 2016.

[30] B. Özkale, R. Parreira, A. Bekdemir, L. Pancaldi, E. Özelçi, C. Amadio, M. Kaynak, F. Stellacci, D. J. Mooney, and M. S. Sakar, "Modular soft robotic microdevices for dexterous biomanipulation," Lab on a Chip, vol. 19, p. 778, 2019. 